\title{
Il "Teatro della memoria" di Mimma Mondadori
}

\section{Sebastiano Martelli}

Non è semplice collocare in un genere letterario questo libro di Mimma Mondadori: ${ }^{1}$ è un romanzo, una biografia, un diario, una storia dell'editoria, una saga familiare alla Dynasty, un raccontodocumento o un racconto memorialistico? E non è neppure facile trovare di conseguenza una chiave di lettura adeguata ed efficace: privilegiare il percorso narrativo, la costruzione del racconto, lasciarsi prendere dal filo della memoria che lega i protagonisti grandi e piccoli della saga familiare Mondadori e dietro questo filo lasciarsi accompagnare da una scrittura controllatissima, sobria $^{2}$ colloquiale quasi da conversazione fabulatoria. ${ }^{3}$ Ma questo percorso di lettura non può essere esclusivo perché oggettivamente ed automaticamente il racconto è anche documento, cronaca, storia di un segmento importante della cultura, dell'industria editoriale e del costume del nostro paese.

Dice l'Autrice nel libro, e lo ha ripetuto anche in molte interviste: "Io racconto i personaggi e non l'epoca. . . . I fatti storici . . . li lascio raccontare da altri. Anche perché sono misteriosi e confusi"; ma in una pagina introduttiva, sorta di epigrafe al volume, sottolinea anche di aver voluto recuperare

certi . . . ricordi e certe suggestioni del passato, di un passato che non è solo il mio, ma quello della mia famiglia, il che vuol dire della Arnoldo Mondadori Editore.

La Mondadori infatti ha rappresentato nel nostro paese per circa un sessantennio un modello imprenditoriale, industriale ed insieme un modello di progettazione, realizzazione e diffusione della cultura: collane e collezioni come Romanica, i Classici, la Medusa, la BMM, gli Oscar, le Enciclopedie, ma anche i Gialli e Topolino, e l'elenco potrebbe essere allungato non poco, tanto è vero che ci sono voluti alcuni anni di lavoro e cinque volumi di 
imminente uscita per mettere insieme il Catalogo storico della Case Editrice.

Una produzione editoriale ed una presenza culturale che hanno attraversato dagli anni Venti fino ai nostri giorni generazioni dopo generazioni di italiani di tutti gli strati sociali. Anzi la forza vitale della Mondadori - come studi recenti hanno evidenziato ${ }^{4}-\mathrm{fu}$ proprio quella di aver capito già negli anni Venti che in Italia stava iniziando una mutazione del pubblico dei lettori, un pubblico nuovo per quantità e qualità che chiedeva prodotti nuovi, cosa che imponeva all'editoria trasformazioni radicali, passando da una dimensione artigianale ad una industriale.

Le vicende di questo primo ventennio della Casa Editrice, sono, come vedremo, piú affidate alla gestione della memoria e del racconto fabulatorio che crea distanza e sfuma sapientemente il documento e la storia, anche se non si trascurano passaggi nevralgici della storia della Casa, come l'appalto della stampa del testo unico delle elementari, cioè del testo di regime che Arnoldo si era assicurato durante il Ventennio. Quando poi nella parte centrale del libro, l'Autrice giunge a trattare degli anni postbellici, della ripresa dell'attività di Mondadori dopo il ritorno dall'esilio volontario in Svizzera a liberazione avvenuta, allora rispunta la testimonianza orgogliosa della figlia che si riconosce nel modello imprenditoriale ed editoriale del padre:

un grande industriale dell'editoria, forse il primo e il più grande. Il panorama editoriale italiano è cambiato grazie a lui.

Nell'Italia degli anni della ricostruzione e del cosiddetto miracolo economico egli

fu l'editore di quella società in sviluppo, ancora una volta il piú sensibile ai bisogni del pubblico e il piú pronto a soddisfarli.

Mio padre è stato l'editore dell'Italia qual era, non ha preteso di inventarne una diversa. Questo era se mai il compito degli editori di cultura, degli editori intellettuali ispirati da una passione politica o ideologica.

Insomma è stato lui a guidare il passaggio ad un'epoca dell'editoria radicalmente nuova, industriale, manageriale, moderna, a portare "chiarezza in un settore fino allora sempre un po' improvvisato e pasticcione," dove imperavano il "dilettantismo" e il "capriccio." Ha dato all'editoria, alla produzione dell'informazione e della cultura una precisa "autonomia industriale" nell'am- 
bito delle regole del profitto cosí come per qualsiasi prodotto, una garanzia per sfuggire al contatto ed agli scopi politici immediati. Arnoldo aveva sintonizzato la produzione libraria e periodica sull'ascolto di una vasta fascia media di fruitori, non confezionando prodotti medî ma creando una fascia mediana di produzione in cui potessero confluire piú strati di lettori, nel senso che potessero trovare prodotti diversificati, da quelli piú popolari a quelli alti per un pubblico piú scelto, piú acculturato o di addetti ai lavori. Sono queste le pagine del libro in cui l'Autrice spinge piú decisamente e chiaramente sull'orgoglio di essere Mondadori e sulla rivendicazione di un' etica industriale in un' Italia che già negli anni della ricostruzione si avviava a conoscere una imprenditorialità cresciuta succhiando alle commesse dello Stato e ad operazioni politico-finanziarie:

Mio padre. . . Era un imprenditore, rischiava, creava, non era uno speculatore o un parassita. Forse oggi siamo in grado di capire un po' meglio questa razza di uomini e di rimpiangere che sia stata poco numerosa. Al loro posto troviamo troppo spesso uomini esperti soltanto di compromessi, di giochi di prestigio e di colpi di mano, incapaci di costruire cose serie che durano.

Sono, queste pagine centrali, quelle piú scoperte sul versante della testimonianza orgogliosa di una figlia, che, dopo le immersioni di impegno diretto nella stessa azienda creata dal padre, propone le proprie convinzioni non solo in quanto figlia e testimone, ma anche come protagonista-continuatrice della stessa attività, sottolineandone la validità del modello. Ma dicevamo della difficoltà di trovare una adeguata chiave di lettura per questo libro: infatti le pagine appena ricordate sono solo piccoli segmenti di una storia dall'interno della famiglia che estrapolate dall'insieme potrebbero dare al lettore l'idea di una testimonianza documentale, di un pamphlet dai toni moderati. Ed invece il libro è tutt'altro.

Forse è utile a questo punto accennare alla genesi di questo racconto, quando e come è nato. Col passare degli anni, si sa, la vita entra in un cono d'ombra particolare in cui le luci che si accendono con piú intensità sono quelle del passato, di ciò che si è vissuto; mentre il tempo si consuma, il futuro - parafrasando il titolo di un famoso libro di Carlo Levi - ha sempre piú "un cuore antico." Ed anche per Mimma Mondadori è maturato ad un certo punto un appuntamento indilazionabile col proprio passato, un dover fare i conti con la storia di se stessa, con le proprie scelte di 
vita, con tutto ciò che si è accumulato dietro di sé; e poi l'esigenza di sistemare il grande flusso dei ricordi, oggettivarli nella scrittura.

Avevo pensato di seguire la strada piú normale - ha dichiarato l'Autrice in un'intervista ${ }^{5}-$ mi ero messa davanti al foglio bianco e, riga per riga, avevo incominciato a stendere le parole una per una. Un'esperienza dura, nel senso che non andavano d'accordo la velocità del ricordo e quella delle mani. Cioè: ricordavo troppo in fretta e scrivevo troppo adagio. Il risultato era una pagina inamidata, senza spessore.

Allora ha deciso di praticare una strada diversa, come per un parto tutto particolare, ha scartato gli strumenti piú comuni: come la madre che accompagna con la sua voce il suo bambino a venir fuori dal buio alla luce del nuovo mondo, della vita, cosí l'Autrice ha scelto la voce per veicolare dalle nebbie del passato e dal mondo sommerso delle memorie il flusso dei ricordi, un passato che chiedeva di venire alla luce. E cosí ha affidato ad un registratore il grande magma di storie, ricordi, immagini, sensazioni: si sono accumulate ventidue bobine sviluppate in circa seicento pagine dattiloscritte, da cui poi ha tratto e rielaborato le duecentodieci pagine del volume a stampa. Questa particolare nascita e costruzione del libro non è un dato esterno, perché in realtà essa segna profondamente la scrittura dandole certe peculiarità, certe qualità che ne fanno una scrittura di confine collocata tra diversi generi: è insomma a questa sua particolare genesi che vanno fatti risalire quel tono colloquiale, quella grande capacità fabulatoria che riesce a trasmettere al lettore senza sforzo alcuno il racconto delle vicende, ma anche il clima di un' epoca, le suggestioni, le immaginazioni, le sensazioni di un mondo e di un tempo; il racconto del passato diventa allora per Mimma Mondadori un "teatro della memoria," per usare un' immagine gombrichiana. ${ }^{6}$

Nella prima metà del libro, quella piú narrativa, il teatro delle memorie è come circondato di luci tenui, di colori delicati, le vicende delle due famiglie d'origine del padre Arnoldo e della madre Andreina, collocate sullo sfondo lontano degli inizi del secolo in un paese del Mantovano, Ostiglia, sono filtrate attraverso i colori sbiaditi del tempo, si ricompongono come in una vecchia foto $d^{\prime}$ epoca ${ }^{7}$ che la scrittura accompagna con tecnica fabulatoria, le vite incrociate di quelli che saranno i protagonisti della storia familiare: "un certo Arnoldo Mondadori, un giovane alle primissime armi come tipografo-editore" e una "bellissima signorina, elegante, un gran cappello all' amor mio non muore' con le rose sotto la testa." La distanza tra i due è notevole: lei raffinata, un 
tantino acculturata, ed anche un po' pasionaria per le sue idee socialiste; lui con idee chiare sulla vita e sul lavoro, una forza vitale prorompente ma da sgrossare, da raffinare culturalmente e socialmente:

Arnoldo e Andreina si fidanzarono a vista e si sposarono di corsa. Mia madre spiegava poi che voleva evadere dalla famiglia e che aveva preso il primo che l'aveva chiesta. A chi le domandava se era innamorata di Arnoldo lei rispondeva che se ne era innamorata dopo. E a me insegnava che questa poteva essere la regola: "ci si innamora dopo."

Rapidi scorci di scrittura che fanno balzare in evidenza la personalità forte dei due maggiori protagonisti del libro, insieme ad Alberto, il fratello della Mimma.

Si snoda cosí il racconto della famiglia Mondadori e della sua ascesa attraverso una attività tipografico-editoriale che la porta a trasferirsi a Verona già nel '19 e poi a Milano: città, paesi, case, le tante ville comprate e lasciate. Ma è all'interno della famiglia che la scrittura entra ad illuminarne con discrezione tutti gli angoli anche quelli piú nascosti, come quelli delle molte infedeltà di Arnoldo, possibili, certamente avvenute ma circondate da una fitta nebbia di mistero e comunque collocabili in quella "morale belle epoque della sua generazione, per la quale moglie e famiglia sono sacre e intangibili, e le avventure galanti soltanto un allegro extra tacitamente concesso all' 'uomo'." Una morale borghese ottocentesca che si riverbera anche nell'educazione dei figli, con una certa separatezza tra il mondo affettivo dei genitori e quello dei figli:

Mia madre era una donna appassionata. I figli, non parlava che dei figli: ma la sua vita era papà, senza di lui il mondo per lei non aveva interesse. Noi figli in modo diverso abbiamo sofferto di gelosia. . . Papà e mamma facevano una vita tutta loro, dalla quale noi eravamo esclusi. Ed era soprattutto lei che avvolgeva papà in una coltre protettiva, lo isolava in una campana di vetro.

Via via nel racconto emerge la forte personalità della madre che amava il marito "in modo esclusivo, con un forte senso di possesso anche fisico e con una capacità di rinuncia a una vita propria che però esigeva in compenso un omaggio assoluto e sempre rinnovato." Per lui voleva essere una moglie e una madre perfetta. Con un sottile ma pungente umorismo l'Autrice segue l'espandersi della personalità materna, il suo occupare e gestire tutti gli spazi possibili all'interno della famiglia fino a plasmare per sé e 
per gli altri autonomamente una immagine ed una personalità dei figli:

Aveva appiccicato un cartello a ciascuno, con le caratteristiche fisiche e morali, e se la realtà a volte non combaciava perfettamente con la descrizione, peggio per la realtà.

Alberto: "la promessa, l'uomo destinato al piú grande successo intellettuale e sociale."

Giorgio: al principio "il bellissimo di casa," poi il figlio "positivo" quello con i "piedi sulla terra," "la persona di cui ci si può fidare," con una sicura etichetta di "solidità."

Mimma: "di animo sereno per definizione, senza alcuna possibilità di essere inquieta o scontenta, aveva anche lei un identikit molto preciso, al quale doveva attenersi: bionda con gli occhi scuri, e se i capelli tendevano a scurirsi come gli occhi, era quasi una disubbidienza e bisognava riparare ossigenandoli." Anche la sua statura era sorvegliata perché non andasse oltre le regole stabilite.

Infine Pucci, l'ultima, la piú piccola, forse proprio per questo con spazi di autonomia maggiore che via via emergono nella storia della famiglia: dalla sua scelta matrimoniale alla sua caparbia voglia di costruirsi una indipendenza anche nel lavoro, prende la licenza liceale insieme con il figlio e si laurea in medicina esercitando anche la professione.

Il teatro delle memorie che Mimma Mondadori viene ricostruendo si illumina delle luci ovattate del Ventennio, ma tutto è ricollocato, rivissuto all'interno del microcosmo familiare. Chi volesse cercare notizie inedite sul rapporto della casa Editrice Mondadori con il Fascismo rimarrebbe deluso; l'Autrice liquida la questione in pochi passaggi:

Mio padre, piuttosto che fascista, era un legalitario: se quello era il governo, lui stava col governo. . .

la madre invece da socialista pasionaria divenne fascista fanatica; ma è anche lei che alla caduta del fascismo, forse con quella intelligenza intuitiva superiore delle donne in certe circostanze, si rende conto che occorre troncare ogni pericoloso prolungamento del passato, e cosí spedisce il marito in Svizzera dove poi sarà raggiunto dal resto della famiglia. 
Tutto, dicevamo, si concentra e si fissa all'interno del gruppo di famiglia: si stagliano sullo sfondo gli interni di case comprate e lasciate senza molti rimpianti perché ciò che conta è altro, le esigenze dell'attività editoriale, e nel racconto le psicologie dei protagonisti, i loro rapporti, l'incrociarsi dei loro destini sono molto piú importanti di ciò che sta intorno. Tutto nel teatro memoriale dell'Autrice si distanzia, si colora, riemerge attraverso multiformi filtri psicologici.

$C^{\prime}$ è una tecnica di scrittura molto efficace nel libro che diventa, specie nella prima parte, il dato stilistico piú pregnante, è una sorta di tecnica della dissolvenza: inserendo dei "credo," "forse," "mi pare," anche i dati storici, cronachistici, realistici sono come distanziati, dissolti nel tempo, circondati di un alone di segni sbiaditi di cui la memoria recupera l'immagine centrale che può essere anche solo un particolare minimo ma è quello che è rimasto, quello che si è accumulato nella memoria della protagonista. Questa tecnica della dissolvenza nella scrittura della Mimma Mondadori è il risultato di una peculiare traduzione di quella parola del ricordo, che, come abbiamo detto, ha avuto una genesi colloquiale attraverso la confessione al magnetofono. Tutto nella distanziazione si carica di struggenti nostalgie e tenerezze, tutto è filtrato attraverso un particolare lessico familiare della memoria in cui la nostalgia ed i possibili sentimentalismi ed idealizzazioni sono riequilibrati dall'ironia, ${ }^{8}$ un'ironia velata ma pungente, che riesce a compensare anche le molte, tante prevedibili reticenze, $\mathrm{i}$ vuoti, i silenzi disseminati lungo la ricostruzione della storia familiare.

Tutto, ha un posto nel teatro della memoria, ma alcuni angoli sono piú illuminati degli altri, non è solo la necessità o la volontà di lasciare poco illuminati certi angoli che produce silenzio e assenza, c'è anche lo sforzo ed insieme la capacità di riandare ed affidarsi alla memoria ed alla psicologia dell'infanzia e dell'adolescenza, tentare di riformulare al lettore la psicologia dell'infanzia e dell'adolescenza con cui lo spazio-tempo della storia della propria famiglia veniva assorbito: ${ }^{9}$ le feste, la cena di Natale, i regali, le presenze-assenze nella casa, i gesti rituali quotidiani

bisogna essere puntuali a tavola. Ho come l'impressione che si andasse a tavola per essere puntuali, non per mangiare.

Le prime uscite e trasgressioni adolescenziali alla "severità sbrigativa" quasi militare imperante in famiglia; e poi il primo flirt, il 
primo bacio "concesso al mio innamorato in ascensore, nel percorso dal pianterreno al sesto piano." Tutto dissolto in poche righe come per il primo matrimonio

conobbi un capo partigiano che aveva al collo un fazzoletto azzurro. Mi disse che era "valdese," e anche questa mi parve una novità sensazionale. Poi si mise al piano e suonò a lungo. Nel mese di novembre del '45 Lo sposai e lasciai la casa paterna. Nel settembre del ' 46 nacque mio figlio Leonardo, e fu come se nascessi finalmente anch'io.

La liberazione del paese sembra coincidere con la liberazione dell'Autrice dai lacci familiari, da quell'educazione che aveva come bloccato la crescita della sua personalità, messa continuamente in angolo da quella dei due genitori: con quel disagio costante alimentato da complessi edipici nei confronti del padre ${ }^{10}$ e quella sottrazione di spazio vitale che avvertiva nei confronti della madre. Ma anche questo è introiettato nel teatro memoriale senza frizioni, immagini e sensazioni le vanno ad ovattare come quei baci e carezze della buona notte, le uniche che la particolare educazione familiare consentisse e che l'Autrice descrive in una pagina di raffinatissima scrittura.

Tutto torna a muoversi nelle luci soffuse del teatro memoriale, dove appaiono e scompaiono, chiamati a sfilare come in un lungo flash back le tante piccole e grandi figure che calcano il palcoscenico di casa Mondadori ed animano un unico, irripetibile spettacolo: lo zio Bruno, la nonna con la sua "passione sfrenata" per le opere liriche, che "cantava, cantava" arie rimaste attaccate alle immagini e sensazioni di quegli anni.

Ma il palcoscenico di casa Mondadori non è solo calcato da figure familiari, com'è prevedibile, pur nella costante separatezza che in particolare la madre tenta sempre di mantenere tra l'attività di Arnoldo e la vita all'interno del nucleo familiare. Sfilano, come in una rapida carrellata di rappresentazioni, i tanti protagonisti della cultura italiana del secolo, una rappresentazione in cui si mescolano pochi dati storici con le immagini assorbite direttamente nel microcosmo familiare, insomma si tratta sempre di profili ricostruiti non attraverso dati esterni di una storia letteraria e culturale pubblica, ma sempre dati alimentati dall'interno del palcoscenico familiare, attraverso i rapporti della famiglia con questi personaggi: da Pastonchi a Pirandello, a D’Annunzio, a Marino Moretti, a Salvator Gotta, Beltramelli, Trilussa, Antongini, Palazzeschi, Alba de Cespedes, Virgilio Brocchi, Lucio D’Ambra, Ales- 
sandro Varaldo, Guelfo Civinini, Italo Balbo. Il tempo che la famiglia trascorre in Svizzera tra la caduta del fascismo e la liberazione è quello piú ricco di notizie, di particolari, imposti forse alla memoria dell'Autrice da uno spazio-tempo di frantumazione dell'unità familiare che impone anche una frantumazione del percorsi esistenziali: è il momento in cui, costretti anche dagli avvenimenti, i quattro figli cominciano a giocare i loro destini in maniera autonoma.

È una prima rifondazione familiare, che avrà un peso non secondario nelle vicende successive non solo della famiglia, ma anche della stessa azienda con i rapporti che nel frattempo Arnoldo ed i figli hanno incominciato a tessere con grandi personaggi della cultura internazionale.

La seconda grande rifondazione avverrà negli anni della ricostruzione, prolungati negli anni del miracolo economico, quando l'azienda entra in un processo di espansione eccezionale, protagonista e lider di una nuova industria editoriale anticipa, segue ed alimenta i nuovi grandi processi di modernizzazione del nostro paese. Ed è qui che il racconto di Mimma Mondadori entra in uno spazio-tempo molto particolare, quello dove si addensano le ombre piú dolorose, quelle piú cariche di amarezze struggenti, di ferite ricomposte ma non rimarginate, di pietas familiare.

La figura di Alberto emerge dal racconto con un protagonismo incontestabile, tanto da essere stato visto da qualche lettore come il vero protagonista del libro. E la scrittura di Mimma riesce con grande capacità evocativa a far vibrare e trasmettere al lettore tutte le corde intime che la legano alla figura del fratello, in un rapporto di "amica e consolatrice." Alberto da sempre si porta dietro il destino di dover essere l'intellettuale della famiglia e la sua vicenda esistenziale sembra come essere la recita di un copione già stabilito, e che egli vive fino in fondo: la sua formazione, le sue amicizie intellettuali con i filosofi milanesi, le lezioni di Banfi e poi i rapporti con Enzo Paci, Anceschi, Cantoni. Una vita sregolata quasi coerente traduzione esistenziale non solo sua ma di un'intera generazione, come Mimma sottolinea:

Il quadro entro cui si svolge la storia di Alberto è quello di tutta una generazione. Avventura estetica, passione politica, cultura nuova. E molto alcool sempre e dovunque: quella fu l'età dell'alcool.

La figura ed il modello dannunziano prima, e poi soprattutto il modello intellettuale ed esistenziale di Hemingway, con cui Al- 
berto ha un lungo e determinante rapporto, costituiscono i referenti, anche se non i soli, entro cui si muove la vicenda di Alberto. Un destino intellettuale ed esistenziale vissuto fino in fondo come in un prestabilito romanzo di una necessaria tranche de vie, che si allarga contestualmente ad una diversa progettualità editoriale e culturale e che converge quasi naturalmente verso l'inevitabile punto di scontro con il padre Arnoldo e la rottura traumatica. Alberto, come è noto, fonderà una sua nuova casa editrice, Il Saggiatore (1967), una esperienza esaltante dal punto di vista culturale, e che nel suo pur breve arco di vita segnerà un momento importante, una operazione di grande prestigio europeo, una delle piú vive di tutta la cultura italiana del Novecento. Dopo due anni l'impresa del Saggiatore viene travolta dalla crisi, altro necessario approdo di un percorso segnato, di una parte stabilita, un rovesciamento di ruolo e di destino rispetto a quello del padre Arnoldo. Dal libro emerge quasi la consapevolezza di entrambi di rappresentare ruoli opposti e inconciliabili, una consapevolezza solcata da un coacervo di amore e rifiuto per il desiderio-impossibilità di essere complementari:

Papà vedeva in Alberto l'uomo di cultura che lui non era. Da parte sua Alberto stimava suo padre perché era l'uomo riuscito, quello che ce l'aveva fatta, un vincitore. Si misurava con lui, voleva uguagliarlo, anzi andare piú avanti.

Sul sottosuolo degli affetti dell'infanzia e dell'adolescenza traumaticamente vissuti nei confronti del padre ma anche della madre, si erano accumulate le frizioni e le differenze, i distacchi, le distanze sempre piú incolmabili, alimentate dalle scelte intellettuali, politiche, esistenziali di Alberto che ormai fuoriuscivano del tutto dal quadro di vita della famiglia d'origine. Ed il destino di Alberto si consuma fino in fondo quasi ad obbedire a quel copione alla Thomas Mann di "salute" e "malattia":

Mio padre e mia madre rappresentavano la "salute" e lui invece la "malattia," loro erano tutta famiglia e lavoro, lui sensibilità, genialità e debolezza.

Intorno a questi lacerti di vita di Alberto, l'Autrice costruisce pagine da "romanzo esistenzialista," "personalità da romanzo esistenzialista" definisce la vita che il fratello si era costruita in quei primi anni Cinquanta tra le sregolatezze di appartamenti pa- 
rigini, cliniche per disintossicarsi dall'alcool ed amicizie con intellettuali famosi. Ma poi l'Autrice, rispettosa dei confini della sua geografia memoriale, risospinge subito la figura del fratello entro i confini e la fa muovere ancora in questa lotta quasi titanica con il padre; di nuovo si ricostruisce la forbice, di nuovo l'ombra di percorsi inconciliabili. Mimma direi che è come una vestale che vorrebbe apprestare tutto il necessario per una cerimonia rituale in un tempio unico per due religioni diverse, è consapevole dell'impossibilità di questo rito unificante ed in fondo lei stessa si sente una vestale scissa tra due religioni diverse:

Arnoldo era un editore industriale, Alberto era un editore intellettuale. L'uno era prima di tutto un imprenditore, l'altro prima di tutto un uomo di cultura e un poeta, e anche il suo modo di vivere e le sue posizioni politiche lo dimostravano.

Come in un romanzo di saga familiare, anche nella storia non romanzata della lotta di lunga durata tra Alberto e Arnoldo solo sul letto di morte del padre tutto sembra ricomporsi: negli abbracci del ritrovarsi con sorelle e parenti nell'anticamera del morente, le lunghe ore ed i giorni che Alberto rimane lí seduto, come ad espiare ed attendere il perdono del Vecchio che nella stanza affianco ha intrapreso l'ultimo estremo viaggio. Cinque anni solo separeranno la sua morte da quella del padre (1971-1976).

Mimma, come una fedele vestale della famiglia, accompagna lo svolgersi dei destini degli altri familiari: la madre, il fratello Giorgio, l'uomo della continuità dell'impresa Mondadori insieme al cognato Mario Formenton, marito della sorella Pucci. L'ultimo capitolo è per la Regina di Casa Mondadori, la madre, accompagnata nei suoi ultimi anni, della malattia, fino alla morte:

La mamma morí a modo suo, com'era vissuta. Imperiosa, capricciosa, dignitosa fino all'ultimo. . .

fedele alla sua immagine di regina fino all'ultimo, di appartata ma forte ingombrante modellatrice del suo e degli altrui destini; anche la morte ne sembra condizionata:

Il suo rapporto con la morte lo definí lei stessa: "Io non ho paura della morte, ho paura di morire." E fu fortunata, perché ebbe la morte senza il morire. Si spense d'improvviso una notte, dopo aver ordinato per l'indomani un piatto di trippa. 


\section{E lei, la Mimma?}

Anche lei, l'Autrice, sembra svolgere fedelmente il suo ruolo di vestale della famiglia; certo anche in lei non può non esserci una fedeltà precaria che conosce le sue ribellioni ed i suoi rifiuti: il primo matrimonio, quasi un atto di liberazione, la sua ricerca di una autonoma collocazione sociale ed economica mettendo su una importante galleria d'arte a Milano, poi l'ingresso nell'attività dell'Azienda Mondadori, la direzione della Fondazione Mondadori.

Ma al di là di queste ed altre scelte di vita, c'è un filo rosso che attraversa tutta la presenza dell'Autrice nella storia della famiglia, ed è questo ruolo di vestale discreta, ruolo accettato fino in fondo:

Io fin dalla piú tenera età sono vissuta nell'ombra della famiglia e della casa editrice, forse perché ero la figlia femmina piú grande, quella che si suppone debba essere la vice della madre. Facevo parte del corredo di famiglia. Sono stata stampata secondo un cliché: casa, libri, Mondadori, moglie, madre. ${ }^{11}$

E come una vestale conserva le memorie di quel grande palcoscenico di casa Mondadori, scandendo i tempi di una intermittente rappresentazione, come per i tanti protagonisti della letteratura italiana di questi ultimi quarant'anni: Bacchelli, figura di "decano" quasi da sempre, uomo di "esprit, fascino e autorevolezza,"

Grande come un vecchio sovrano, un giorno a casa mia si incastrò in una poltrona, e ci dovemmo mettere in tre per tirarlo su: due tenevano ferma la poltrona, il terzo lo tirava puntando i piedi, e lui si lasciava fare con aria imperturbabile.

\section{Silone}

un uomo che portava scritti sulla faccia la lealtà, il coraggio, una fermezza invincibile e insieme una modestia senza finzione. Aveva un garbo infinito, ma era chiuso come una conchiglia.

D'Arrigo, che cammina con le "sue inseparabili scarpette d'aereo" sopra "bozze e manoscritti chilometrici," "una delle poche persone in cui ho sentito il genio." Piero Chiara, con la sua "solita intelligenza penetrante e ironica." Diego Valeri, "uomo adorabile, stupendo, vecchio elegante, affettuoso, dai modi insieme eleganti e familiari." Montale a "tratti divertentissimo" ma che "intimidiva per la sua grandezza." E poi Buzzati, "di una timidezza assoluta"; molto suggestiva l'immagine che l'Autrice ci dà 
di Buzzati accanto al letto di morte del vecchio Mondadori, quando forse sapeva già di dover morire per il male che lo incalzava: "era il viso dell'uomo che si specchia e cerca di capire." Quasi, aggiungerei, a scrutare sul volto del vecchio Arnoldo i segni di una appartenenza allo stesso "reggimento," quel "reggimento" - eccezionale ed originale metafora di una morte annunciata - che ormai si è messo in movimento e sente che è il suo stesso reggimento. Scriverà Buzzati in un racconto scritto poco prima della morte:

tutti in certo modo appartengono a un reggimento e i reggimenti sono innumerevoli, nessuno sa quanti sono, e nessuno sa neanche quale sia il suo reggimento, eppure i reggimenti sono accantonati qui intorno, anche nel cuore della città, benché nessuno se ne accorga e ci pensi. Però quando un reggimento parte, chi gli appartiene, pure lui deve partire. ${ }^{12}$

Sul palcoscenico allestito da Mimma Mondadori sfilano altri personaggi non senza una scelta alquanto selettiva, un po' per necessità un po' per altre ragioni, poiché in fondo non sono tanti quelli fatti sfilare rispetto ai molti piccoli e grandi che hanno calcato le scene di quel palcoscenico e che comunque sono entrati in quell'ininterrotto spettacolo rappresentato dentro e fuori la Casa Editrice. Compaiono Guido Piovene, Gianna Manzini, il "nume tutelare" Carlo Bo, Enzo Biagi, Indro Montanelli di cui l'Autrice si innamorò a quattordici anni e poi amico continuamente perso e ritrovato. Ed ancora Vittorio G. Rossi, Tobino, Soldati, Vittorio Sereni, Bassani con i suoi tic, i suoi capricci, i suoi snobismi. E la vestale Mimma sembra tutto accettare comprendere e ricomporre nel suo teatro memoriale distendendovi sopra, quando è necessario, un velo d'ironia, come nella descrizione della cena in occasione del Premio Campiello vinto da Tobino, quando non era semplice tentare di evitare la prevedibile suscettibilità dei vari Piovene, Soldati, Bassani, Neri Pozza, Spadolini, per l'assegnazione dei posti a tavola. Sull'episodio l'Autrice stende alla fine come un velo sottile carico di lievissima ironia mista alla struggente nostalgia e rimpianto per un altro spazio-tempo del suo villaggio memoriale:

Ricordo questo filmetto accelerato e un po' comico e sorrido con malinconia pensando che se ci fosse stato papà la scena si sarebbe svolta invece in un tempo largo maestoso, anzi: largo maestoso con bonomia. 
Ma il grande sacerdote ormai non c'è piú: a lei, alla affettuosa discreta vestale non resta che immaginarlo, come in un lungo sogno ininterrotto, quasi prolungamento necessario della vita, alle prese con una tipografia in paradiso, come le aveva sussurrato sul letto di morte prima di intraprendere l'ultimo estremo viaggio:

"Sono sicuro, sai," mi diceva "di là c'è sicuramente una tipografia. Se le cose sono giuste io trovo la mia tipografia."

\section{Università di Salerno}

\section{NOTE}

1 M. Mondadori, Una tipografia in paradiso (Milano: Mondadori, 1985).

2 Cfr. G. Pacchiano, "La classe editrice va in paradiso," in L'Europeo (26 ottobre 1985).

3 "Un notevole volume memorialistico" l'ha definito Gianfranco Contini (Intervista rilasciata a F. Contorbia, in La Stampa-Tuttolibri [28 settembre 1983]).

4 AA. VV., Editoria e cultura a Milano tra le due guerre (1920-1940), Atti del Convegno: Milano, 19-21 febbraio 1981 (Milano: Fondazione Arnoldo e Alberto Mondadori, 1983).

5 M. Mascardi, "Casa Mondadori: cronaca di un amore," in Grazia (29 settembre 1985).

6 Cfr. H. Gombrich, Custodi della memoria (Milano: Feltrinelli, 1985).

7 "Gli 'inizi' del racconto sono fiabeschi, tipo 'c'era una volta.' Non solo Arnoldo e Andreina giovani sembrano i fantasmi di una favola, ma tutto il mondo che sta loro intorno è una favola. E non si tratta di magia da parte della narratrice, ma della storia ... il nostro Paese era tutto una favola, remoto, artigianale, arcaico, carico di fermenti e di intelligenza . . .": C. Marabini, "La cultura italiana alla corte di papà," in il Resto del Carlino (18 ottobre 1985).

8 La funzione equilibratrice dell'ironia è stata sottolineata tra gli altri anche da: O. Del Buono, "Mondadori una saga in tipografia," in La Stampa-Tuttolibri (14 settembre 1985); C. Castellaneta, in Oggi (2 ottobre 1985).

9 La storia della famiglia è narrata "con uno straordinario senso della quotidianità del bozzolo familiare": L. Vergani, "Gruppo di famiglia in una tipografia," in Corriere della Sera (16 settembre 1985).

10 "Con papà . . . avevo il mio bel Edipone, il mio complesso di Edipo. . . . L'ho amato, sono stata fiera di lui. Sempre. Non solo da ragazzina": Intervista rilasciata a W. Valli, in Il Lavoro (10 ottobre 1985).

11 ". . . a questa sorta di memoria del proprio clan attinge sempre con discrezione e misura ... a lei compete soltanto quella posizione apparentemente decentrata, ridotta alle cose che ha visto, ai sentimenti che l'hanno nutrita e protetta": R. Minore, "I ruggiti del patriarca," in Il Messaggero (20 ottobre 1985).

12 D. Buzzati, Il reggimento parte all'alba (Milano: Frassinelli, 1985), p. 2. 